\title{
Can p53 expression and staining intensity correlate with histopathological prognostic parameter and clinical staging in head and neck squamous cell carcinoma?
}

\author{
Kaur Jasmeet ${ }^{1}$, Mannan Rahul ${ }^{1}$, Manjari Mridu ${ }^{1}$, Sharma Sonam², \\ Kaur Jasmine ${ }^{3}$, Bhasin Tejinder Singh ${ }^{1}$, Kaur Amritpal ${ }^{1}$ \\ ${ }^{\prime}$ Department of Pathology, Sri Guru Ram Das Institute of Medical Sciences and Research, Amritsar, Punjab, India \\ ${ }^{2}$ Department of Pathology, Kalpana Chawla Government Medical College, Karnal, Haryana, India \\ ${ }^{3}$ Department of Oral and Maxillo-Facial Surgery, Sri Guru Ram Das Institute of Dental Sciences and Research, Amritsar, Punjab, India
}

\author{
Keywords: \\ Head and Neck; \\ p53; \\ Immunoexpression; \\ Intensity; \\ Squamous Cell \\ Carcinoma;
}

\begin{abstract}
Background: : In recent years, p53 has emerged as an important tool for not only diagnosis and predicting prognosis in head and neck squamous cell carcinoma. The aim of this study was to find the role of p53 staining intensity in determining prognosis.
\end{abstract}

Materials and Methods: Fifty histopathologically proven cases of squamous cell carcinoma of head and neck were studied. The findings of the study were analyzed particularly in reference to p53 expression and their correlation with age, sex, anatomical site, tumor size, histological grading, vascular, peri-neural, muscle invasion, lymph node metastasis and staining intensity.

Results: Immunopositivity rate of p53 was $64 \%$ with percentage positive cells varying from 5-76\% with mild, moderate and strong staining intensity. A positive correlation of p53 independently was seen with oral cavity, grade, lymph node metastasis and pathological staging.

Conclusion: p53 immunoexpression is an important independent variable of prognostication.

\section{Correspondence:}

Dr. Sonam Sharma, MBBS, MD

Assistant Professor, Department of Pathology

Kalpana Chawla Government Medical College, Karnal, Haryana, India. ORCID ID: 0000-0001-9856-9542

Email: drsonamsharma@gmail.com

Received : May 27th, 2017 ; Accepted : August 3rd, 2017; Published : September 1, 2017

Citation: Kaur J, Mannan R, Manjari M et al. Can p53 expression and staining intensity correlate with histopathological prognostic parameter and clinical staging in head and neck squamous cell carcinoma? Experience of a small Indian cohort. J Pathol Nep. 2017;7:1141-8. doi: 10.3126/jpn. v7i2.17976

Copyright: This is an open-access article distributed under the terms of the Creative Commons Attribution 4.0 International License, which permits unrestricted use, distribution, and reproduction in any medium, provided the original author and source are credited.

\section{INTRODUCTION}

Head and neck squamous cell carcinoma (HNSCC) is the sixth most common cancer in the world. 5,00,000 new cases are reported every year worldwide and are associated with increased morbidity and poor mortality indices with a poor five-year survival rate of $40-50 \% .{ }^{1}$ Overall they account for about $3 \%$ of human cancers with about $90 \%$ of cancers localised in oral cavity. ${ }^{2}$

In India, HNSCC is a major form of cancer accounting for $23 \%$ of all cancer in males and $6 \%$ in females with an estimated 2.5 lakh new patients diagnosed every year, of whom about three-fourths are in an advanced stage., Most of the cases in Indian population arise from oral cavity and tongue unlike pharyngeal and/or laryngeal SCC seen as in Asian, European and American population 
studies. Increased tobacco (chewing and smoking), alcohol consumption and increased prevalence of infection with high-risk types of human papilloma virus (HPV) are the major risk factors. ${ }^{5,6}$ The most common pathophysiology behind its occurrence has been attributed to the concept of "field-cancerization", in which there is increased risk of cancer development in the entire head and neck region due to multiple genetic abnormalities after prolonged exposure to carcinogens. The numerous independent foci of abnormal tissue that are obtained after mutations gives rise to premalignant/potentially malignant dysplastic lesions, from which usually these invasive carcinomas develop. ${ }^{7}$ These dysplastic lesions include leukoplakia, oral sub mucous fibrosis and erythroplakia etc. all of which are more prevalent in India due to poor oral hygiene, consumption of spicy food, alcohol and an acceptable societal norm of betel quid/ areca nut/ tobacco chewing. ${ }^{1}$

The various modalities used for its diagnosis are endoscopy, imaging studies including X-rays, computed tomography (CT), magnetic resonance imaging (MRI) or positron emission tomography (PET) scans, histopathological examination, Immunohistochemistry (IHC) and molecular markers.

In recent years, IHC in HNSCC has emerged as an important tool for diagnosis and predicting prognosis. Various IHC markers are used as a research tool in cases of HNSCC such as - p53, Ki-67, CD-44, CD-133, and c-met. These markers have also helped treating physicians for proper therapeutic stratifications. p53 also known as TP53 or tumor protein (EC:2.7.1.37) is a gene that codes for a protein that regulates the cell cycle and is a wellknown tumor suppressor gene reported to have central role in various human neoplasia such as colon, breast, lung, prostate, oesophagus, sarcomas, hematological malignancies, brain tumor and also associated with Li-Fraumeni and related syndromes. The $\mathrm{p} 53$ protein is a DNA-binding protein product of the p53 tumor suppressor gene encompassing $16-20 \mathrm{~kb}$ of DNA on the short arm of chromosome 17 at position 17-13.1. Wild-type p53 protein exerts growth-inhibitory activity. Its up-regulation in human hematopoietic cells in response to certain types of DNA damage prolongs the G1 phase of the cell cycle and gives the cell time to repair that damage before entering the $\mathrm{S}$ phase. ${ }^{8}$ Hence, overall $\mathrm{p} 53$ plays a key role in mediating cell response to various stresses, mainly by inducing or repressing a number of genes involved in cell cycle arrest, senescence, apoptosis, DNA repair and angiogenesis.
Although there are studies done in Indian sub-continent, describing the relationship between squamous cell carcinoma in oral cavity with p53, but, not many are available describing the relationship of p53 in whole of head and neck region. Also, very few have tried to investigate HNSCC with various clinicopathological parameters as well as with score (staining intensity).

The present study was conducted at a tertiary care teaching hospital of North India catering to predominantly rural based Punjabi population. The lesions were classified and graded on histomorphology and expression of p53 was noted on immunohistochemical staining. The findings of the study were analyzed particularly in reference to p53 expression and their correlation with age, sex, anatomical site, tumor size, histological grading, vascular, perineural, muscle invasion, lymph node metastasis and staining intensity (final score).

\section{MATERIALS AND METHODS}

The study was conducted on 50 cases of HNSCC diagnosed at the Department of Pathology, Sri Guru Ram Das Institute of Medical Sciences and Research, Amritsar, Punjab, India. Prior to the research, permission was obtained from institutional review committee. Of these 50 cases of SCC; 28 cases were composite radical specimens (mandibulectomy, maxillectomy, glossectomy or laryngectomy) with both side modified/radical neck dissections. Rest 22 cases of $\mathrm{SCC}$ were punch/excision biopsies from various anatomical sites of head and neck region. Detailed clinical data of the patient was recorded. Histopathological examination of the tissues obtained was done after processing them to prepare paraffin blocks, followed by staining with stained with Haematoxylin and Eosin stain. The slides were studied under light microscopy for histopathological grading and to document other pathological parameters (histological grading, vascular, perineural, muscle invasion, lymph node metastasis).

Immunohistochemistry was done on formalin-fixed sections, and paraffin embedded 2-4 $\mu \mathrm{m}$ sections of representative blocks of each tumor were mounted on poly-lysine precoated slides. Antigen retrieval was done in a pressure cooker using citrate buffer solution at $\mathrm{pH}$ 6.0. Peroxidase inhibition was then done, followed by washing in tris buffer saline and incubation in protein block. To evaluate p53 expression, p53 antibody (Diagnostic Biosystem) was used. Post primary block was then applied and incubation

Table 1: p53 expression at different anatomical sites

\begin{tabular}{lcccl}
\hline p53 & Oral Cavity/Oropharynx & Larynx/Hypopharynx & Nasopharynx & Total (\%) \\
\hline Positive & $28(70 \%)$ & $03(37 \%)$ & $01(50 \%)$ & $32(64)$ \\
Negative & $12(30 \%)$ & $05(63 \%)$ & $01(50 \%)$ & $18(36)$ \\
\hline Total & $\mathbf{4 0}$ & $\mathbf{0 8}$ & $\mathbf{0 2}$ & $\mathbf{5 0}(\mathbf{1 0 0})$ \\
\hline
\end{tabular}


Table 2: Correlation of $\mathrm{p} 53$ status with age and gender

\begin{tabular}{lccc}
\hline \multirow{2}{*}{$\begin{array}{l}\text { Age Group } \\
\text { (yrs) }\end{array}$} & \multicolumn{2}{c}{$\mathbf{p 5 3}$ expression } & Total \\
\cline { 2 - 3 } & Positive & Negative & \\
\hline $\mathbf{2 1 - 4 0}$ & 06 & 01 & 07 \\
\hline $\mathbf{4 1 - 6 0}$ & 16 & 13 & 29 \\
\hline $\mathbf{6 1 - 8 0}$ & 10 & 04 & 14 \\
\hline Total & $\mathbf{3 2}$ & $\mathbf{1 8}$ & $\mathbf{5 0}$ \\
\hline
\end{tabular}

was done with $\mathrm{DAB}$ (3,3'diaminobenzidine). Sections were washed in deionised water followed by Haematoxylin counterstaining, dehydration and clearing of the sections in propanol and xylene respectively. Antigen thus expressed was visible under light microscopy as brown coloured nuclei of variable staining intensity.

\section{Inclusion Criteria}

Purely SCC were included in the study. Only the cases which had more than $5 \%$ cells positive for p53 were included for final scoring. Positive control was colonic cancer cases which were positive for $\mathrm{p} 53$. Negative control section was provided by omission of primary antibody. Brown nuclei were taken as positive for $\mathrm{p} 53$.

The intensity of p53 positivity was scored as - No staining $(0)$, weak staining $(+1)$, moderate staining $(+2)$ and intense staining $(+3)$. The final score ( $\mathrm{H}$ score) was then calculated by multiplying the intensity of the staining score with proportional positivity score. The proportional positivity score were 0 (if $<5 \%$ nuclei expressed), 1 (5-25\% nuclei expressed), 2 (25-50\% nuclei expressed) and 3 (if $<50 \%$ nuclei expressed).

Parameters included were age, sex, anatomical site, tumor size, histological grading, vascular, perineural, muscle invasion, lymph node metastasis and staining intensity (final score).

\section{Statistical Analysis}

The relationships between IHC expression and various clinicopathological parameters were statistically analyzed using SPSS version 12 software. Chi-square test was used for data analysis. $\mathrm{p}<0.05$ was considered statistically significant.

\section{RESULTS}

The commonest site involved in the present study was oral cavity and oropharynx (80\%) followed by hypopharynx and larynx (16\%). The least common site was nasopharynx (4\%). Most of the patients presented with complaints of difficulty in speaking, dysphagia and complained of nonhealing ulcer.
Table 3: Correlation of p53 expression with tumor size

\begin{tabular}{lccc}
\hline \multirow{2}{*}{$\begin{array}{l}\text { Tumour } \\
\text { Size }\end{array}$} & \multicolumn{2}{c}{ p53 expression } & \multirow{2}{*}{ Total } \\
\cline { 2 - 3 } & Positive & Negative & \\
\hline pT1 & 00 & 00 & 00 \\
\hline pT2 & 05 & 07 & 12 \\
\hline pT3 & 02 & 00 & 02 \\
\hline pT 4 & 09 & 05 & 14 \\
\hline Total & $\mathbf{1 6}$ & $\mathbf{1 2}$ & $\mathbf{2 8}$ \\
\hline
\end{tabular}

Table 4: Correlation of p53 expression with grade of carcinoma

\begin{tabular}{lccc}
\hline \multirow{2}{*}{ Grade } & \multicolumn{2}{c}{$\mathbf{p 5 3}$ expression } & \multirow{2}{*}{ Total } \\
\cline { 2 - 3 } & Positive & Negative & \\
\hline Well & 02 & 08 & 10 \\
\hline Moderate & 23 & 09 & 32 \\
\hline Poor & 07 & 01 & 08 \\
\hline Total & $\mathbf{3 2}$ & $\mathbf{1 8}$ & $\mathbf{5 0}$ \\
\hline
\end{tabular}

\section{p53 Immunoexpression}

Overall, immunopositivity rate of p53 was $64 \%$ of all the HNSCC cases, while subdividing the head and neck region site wise, majority of SCC of oral cavity showed immunopositivity (70\%). In nasopharynx region, only half of the cases showed p53 immunopositivity (50\%) while in laryngeal SCC, one third of the cases showed immunopositivity (37\%). Percentage positive cells varied from $5-76 \%$ with mild, moderate and strong staining intensity. Thus, SCC of oral cavity and oropharyngeal region showed a strong expression for $\mathrm{p} 53$, while the immunopositivity rates were low in laryngeal and nasopharyngeal region. (Table 1)

Age group specific and gender specific p53 Immunoexpression correlation. The majority of patients included were in age group of 41-60 years (58\%) with male: female ratio being 7.3:1.

On correlating the $\mathrm{p} 53$ expression with age specific groups, it showed highest positivity (50\%) in the patients above 40 years of age. However, no significant correlation between age group and gender with p53 expression was seen statistically ( $\mathrm{p}$ value being 0.253 and 0.684 respectively). (Table 2)

\section{Correlation of p53 Immunopositivity with Pathological Staging.}

In 26 cases the pathological staging was possible which were composite specimen; according to the tumor size and lymph node metastasis. Majority of the cases were stage 4 (pT4) followed by stage 3 (pT3) and stage 2 (pT2). p53 immunopositivity in pT4, pT2 and pT3 category was $78.5 \%$, 
Table 5: Correlation of p53 final score with grade of carcinoma

\begin{tabular}{|c|c|c|c|c|}
\hline p53 & Oral Cavity Oropharynx & Larynx/Hypopharynx & Nasopharynx & Total (\%) \\
\hline $\mathbf{0}$ & 08 & 09 & 01 & 18 \\
\hline 1 & 02 & 02 & 00 & 04 \\
\hline 2 & 00 & 10 & 01 & 11 \\
\hline 3 & 00 & 02 & 02 & 04 \\
\hline 4 & 00 & 03 & 00 & 03 \\
\hline 6 & 00 & 03 & 03 & 06 \\
\hline 9 & 00 & 03 & 01 & 04 \\
\hline Total & 10 & 32 & 08 & 50 \\
\hline
\end{tabular}

$12.5 \%$ and $80 \%$ respectively. Hence, it was noted that with an increase in stage, p53 expression increases which was found to be statistically significant ( $p=0.009$, Chi square test). (Table 3)

\section{Correlation of p53 Immunoexpression and Tumor Grade}

Most of the cases included in this study were moderately differentiated constituting $64 \%$ of the total cases, followed by well and poorly differentiated tumors constituting $20 \%$ and $16 \%$ respectively(fig. 1a, $1 \mathrm{c} \& 2 \mathrm{a}$ ).

Correlating tumor grade with p53 expression, it was noted that in well differentiated carcinomas, the rate of expression was $20 \%$, in moderately differentiated, the immunoexpression rate was higher (72\%) and in poorly differentiated carcinomas, immunoexpression rate was highest (88\%). Thus, an increase in p53 immunopositivity expression was observed with increase in tumor grade, and this was found to be statistically significant ( $p=0.004$; chi square test). (Table 4)

\section{Correlation of p53 score (stain intensity) with Grade of Carcinoma}

All the well-differentiated cases had lower scores $(0$ and $1)$. Half of the poorly differentiated cases $(50 \%)$ had higher scores of 8 and 9 (fig. 1b, 1d, 2b). A significant correlation between grade of carcinoma and p53 final score was seen $(\mathrm{p}<0.05$; chi square test). (Table 5$)$

\section{Correlation of p53 expression with Lymph node Status}

Out of the 28 cases of composite radical specimens with bilateral neck dissection, lymph nodes of variable sizes were recovered in 26 cases only. Metastasis was seen in 15 cases, of which $\mathrm{p} 53$ positivity was seen in two-third of the cases(11/15;73\%). In the remaining 11 cases where lymph nodes were reactive (absence of carcinomatous deposits); the p53 expression was low $(3 / 11 ; 27 \%)$. Hence, a relationship in the present study proposes that $\mathrm{p} 53$ positive cases have an
Table 6: Correlation of p53 expression with lymphnode status

\begin{tabular}{lccc}
\hline \multirow{2}{*}{$\begin{array}{l}\text { p53 } \\
\text { expression }\end{array}$} & \multicolumn{2}{c}{ Lymph node status } & \multirow{2}{*}{ Total } \\
\cline { 2 - 3 } & Metastatic & Reactive & \\
\hline Present & 11 & 03 & 14 \\
\hline Absent & 04 & 08 & 12 \\
\hline Total & $\mathbf{1 5}$ & $\mathbf{1 1}$ & $\mathbf{2 6}$ \\
\hline
\end{tabular}

increased propensity to metastasize to regional lymph nodes and this was found to be statistically significant. ( $\mathrm{p}$ value $=$ 0.0204). (Table 6)

\section{Correlation of p53 expression with Vascular, Perineural and Muscle Invasion}

Out of the 28 specimens received, 11 (39\%) showed vascular invasion, 2 cases (7\%) showed perineural invasion and muscle invasion was seen in 14 cases $(50 \%)$.

p53 positivity was seen in $54.5 \%$ cases with vascular invasion and $64 \%$ cases with muscle invasion. Both cases showing perineural invasion showed p53 positivity. No significant correlation of p53 expression with vascular, perineural and muscle invasion was found with $\mathrm{p}$ values of $0.823,0.204$ and 0.445 respectively (Chi square test). (Table 7)

\section{DISCUSSION}

The incidence of SCC in head and neck region is on a dramatic increase in India since early eighties. More and more cases are being diagnosed at an early stage due to increased awareness and better diagnostic modalities. Of these; Oral cancer is reported to be the most frequent malignant tumor of head and neck region worldwide and India as well. ${ }^{1,3}$

In this scenario, a simple diagnosis of SCC especially on punch biopsies and dysplastic lesions, is not sufficient for further management and accurate as well as economically 
Table 7: Correlation of p53 expression with vascular, perineural and muscle invasion

\begin{tabular}{lcccccc}
\hline \multirow{2}{*}{ p53 expression } & \multicolumn{5}{c}{ Lymph node status } \\
\cline { 2 - 7 } & \multicolumn{3}{c}{ Vascular } & \multicolumn{2}{c}{ perineural } & Muscle \\
\cline { 2 - 7 } & Present & Absent & Present & Absent & Present & Absent \\
\hline Positive & 06 & 10 & 02 & 13 & 09 & 07 \\
Negative & 05 & 07 & 00 & 13 & 05 & 07 \\
\hline Total & $\mathbf{1 1}$ & $\mathbf{1 7}$ & $\mathbf{0 2}$ & $\mathbf{2 6}$ & $\mathbf{1 4}$ & $\mathbf{1 4}$ \\
\hline
\end{tabular}

viable methods (having a good cost benefit ratio) to predict prognosis and eventually to determine appropriate therapy is the need of the hour in third world countries like ours. In radical surgeries, we can predict to a relative degree, prognosis on the basis of lymph node metastasis recovered with the specimen. But, the same is not so in the small punch biopsies for various head and neck regions, as only small volume of tumor is sampled in punch biopsies leading to often under sampling of the tumour tissue. In such cases, newer histopathological parameters (which should be included in the light microscopic report) and molecular markers can predict presence of higher grade areas which might be missed in sampling because molecular discrepancies leading to neoplasia often precede eventual histopathological changes.

In developed countries, techniques such as FISH, RT-PCR, glycolytic pathways are being increasingly used for better diagnostic, prognostic and therapeutic purposes in the field of HNSCC. But, in many resource challenged countries of Asia and Africa, these facilities are not widely available. Here, detailed clinicohistomorphological parameters and a single inexpensive IHC marker can play a major role in sub planting aforementioned ancillary diagnostic tools for better patient management and prognostication.

p53 has been described as "the guardian of the genome", referring to its role in conserving stability by preventing genome mutation. p53 was identified in 1979 by Arnold Levine, David Lane and William Old, working at Princeton University, Dundee University and Sloan-Kettering Memorial Hospital respectively. The human p53 gene is located on the seventeenth chromosome (17p13.1).

It plays an important role in cell cycle control and apoptosis. In normal cells, the p53 protein level is low. DNA damage and other stress signals may trigger the increase of p53 proteins which have three major functions: growth arrest, DNA repair and apoptosis (cell death). The growth arrest stops the progression of cell cycle preventing replication of damaged DNA. During the growth arrest p53 may activate the transcription of proteins involved in DNA repair. Apoptosis is the "last resort" to avoid proliferation of cells containing abnormal DNA.

If p53 gene is damaged, tumour suppression is severely reduced. Defective p53 could allow abnormal cells to proliferate resulting in cancer. People who inherit only one functional copy of p53 will most likely develop tumors in early adulthood, a disease known as Li-Fraumeni syndrome. p53 can also be damaged in cells by mutagens (chemicals, radiation or viruses) increasing the likelihood that the cell will begin uncontrolled division.

Taneja $\mathrm{K}$ et al, did a research to demonstrate the expression of p53 in cases of HNSCC and found the frequency of p53 expression to be associated with tumor histological grade, increasing lymph node involvement and clinical stage $(\mathrm{p}=$ 0.038). ${ }^{9}$ Boslooper $\mathrm{K}$ et al demonstrated that $\mathrm{p} 53$ expression was related to higher grades of HNSCC and a high portion of tumor cells expressing p53 had a shorter survival than the other groups. ${ }^{10}$

The purpose of present study was to determine the expression of p53 in cases of HNSCC and to determine the relationship of p53 expression and intensity with various clinicohistomorphological parameters. In the present study, 50 cases, both punch biopsies and radical specimens were taken up. These were graded and compared with various epidemiological parameters like anatomical site, parameters of tumour aggressiveness (in the form of pathological staging, invasion and lymph node metastasis.

Most of the patients in the present study were in the age group of 41-60 years of age with higher male preponderance showing that HNSCC usually affects elderly age group and is seen mainly in males. This is in corroboration with the work done by other reseachers. ${ }^{2,11}$ No significant correlation was elicited between age as well as gender and p53 expression. Many researchers like Dragomir LP, Boslooper $\mathrm{K}$, Geisler SA and Li Y had also concluded the same., ${ }^{2,10,12,13}$

In the current study, p53 positivity was seen in $64 \%$ of the cases (percentage positive cells varying from $5-76 \%$ with mild, moderate and strong staining intensity and final score varying from 0 to 9 ). Similar results were reflected in the work done by Taneja $\mathrm{K}$ et al and Boslooper $\mathrm{K}$ et al who had reported a p53 expression as $60 \%$ and $63 \%$ positivity respectively. ${ }^{9,10}$ A high immunoexpression of p53 in the oral cavity and oropharynx (70\%) is in corroboration with the work conducted by Dragomir LP et al, Jain A et al and Kerdpon D et al who reported a p53 expression in oral cavity SCC as $82.3 \%, 74.3 \%$ and $94 \%$ respectively. ${ }^{2,14,15} \mathrm{~A}$ p53 positivity of $50 \%$ in nasal cavity and nasopharynx 


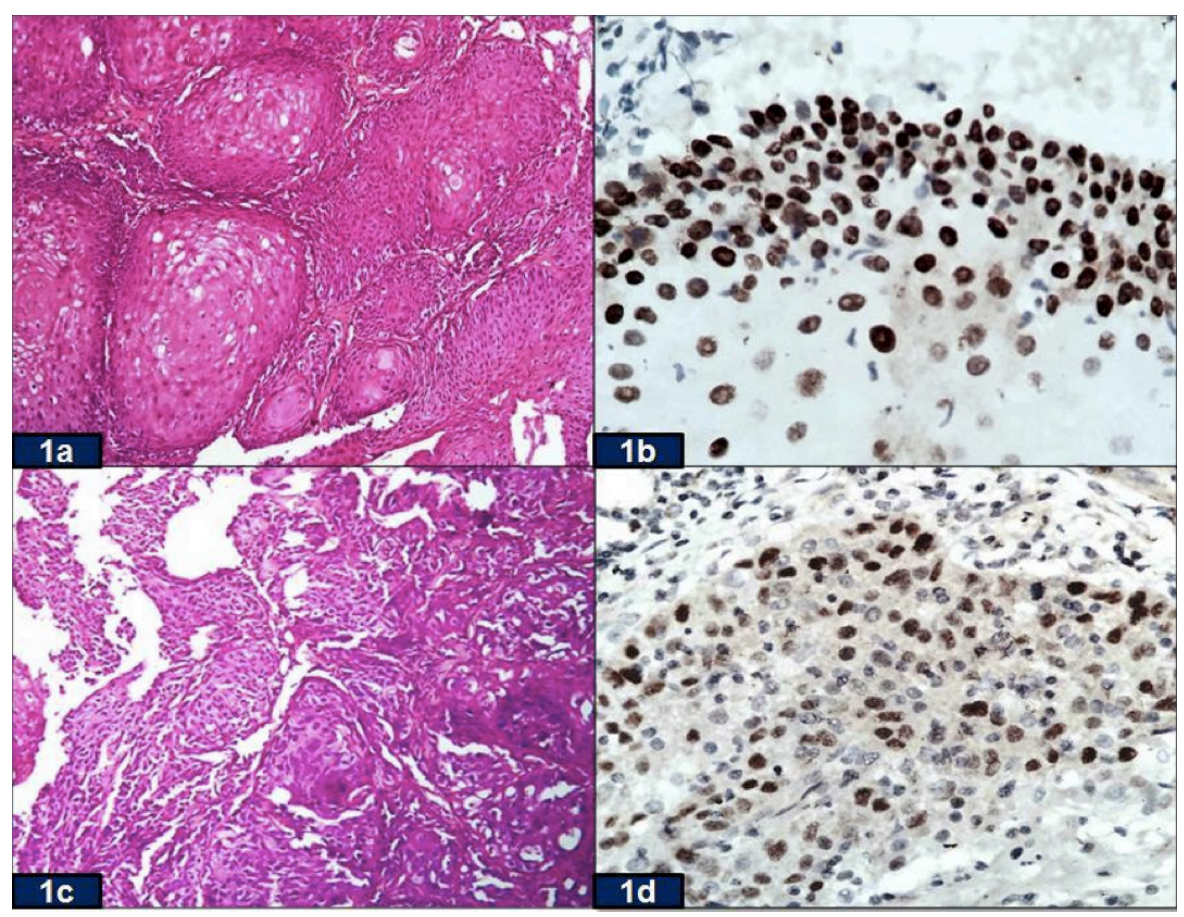

Figure1: a) Well differentiated squamous cell carcinoma (HE stain; X 200). b) Tumor cells exhibiting 553 expression of +3 intensity (IHC stain; X 400). c) Moderately differentiated squamous cell carcinoma (HE stain; X 200). d) Tumor cells showing +2 intensity p53 expression (IHC stain; X 400).

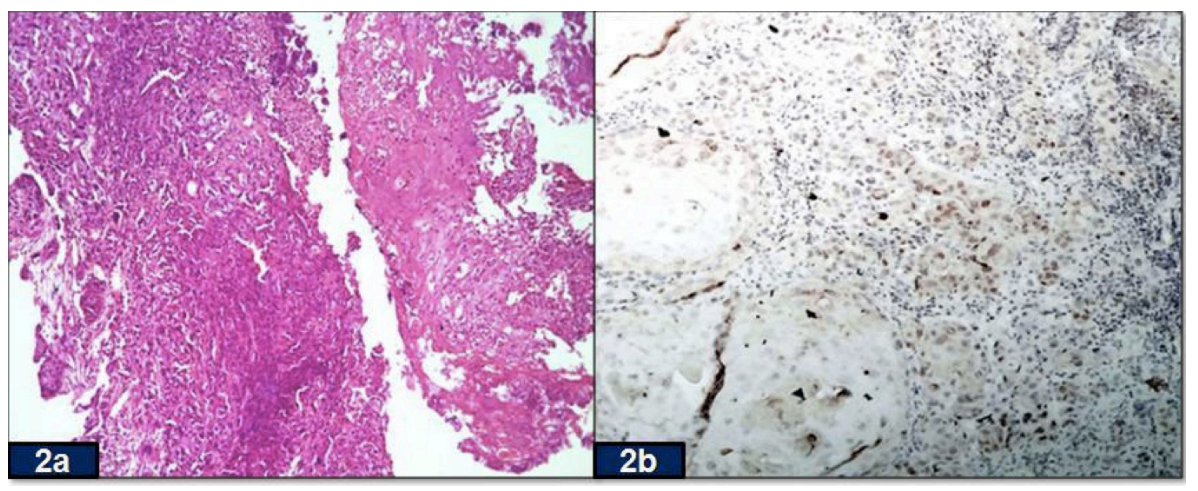

Figure 2: a) Poorly differentiated squamous cell carcinoma (HE stain; X 200). b) Tumor cells exhibiting weak (+1) p53 staining intensity (IHC stain; X 100).

finds coherence with the work conducted by Franzman MB et al which has reported an immunoexpression of around 60 percent. $^{8}$ The p53 positivity in cases of larynx and hypopharynx was relatively low (37\%). This is in contrast to a study conducted by Ashraf MJ et al who had reported a much higher expression rate of $64.8 \%$ in SCC of larynx. ${ }^{10}$

In the present study, a correlation was seen between $\mathrm{p} 53$ expression and tumor grade. $88 \%$ cases having poorly differentiated carcinoma showed positivity with p53 in comparison to $72 \%$ in moderately and $20 \%$ in well differentiated carcinomas. An increase in p53 expression was seen with increase in tumor grade and was found to be statistically significant $(p=0.004)$. This correlation was also seen in the lesions of oral cavity and oropharynx ( $p$ $=0.001$ ) but not in nasopharyngeal and laryngeal cases. These findings find corroboration with the work done by various researchers in HNSCC and OSCC., ${ }^{9,10,14}$ However, many studies have shown no correlation with the degree of differentiation in oral cancers. ${ }^{2}$ In contrast to our study, Ashraf MJ et al found a correlation between p53 expression and tumor grade in laryngeal SCC. ${ }^{17}$ When the p53 immunointensity was correlated with tumor grade, it was found that as grade increases, intensity of p53 stain increased. This was also found to be statistically significant ( $p=0.011$, chi square test). This implied that both immunoexpression as well as intensity of staining are independent variables in predicting eventual tumor 
grading, as a non representative punch biopsy if exhibiting immunoexpression and increased intensity in dysplastic/insitu component or in tumour tissues will be associated with a poor prognosis.

While assessing the invasiveness of HNSCC with p53 expression, it was found that $\mathrm{p} 53$ positivity was seen in $73 \%$ of metastatic cases but only $27 \%$ in case of reactive nodes. This positive correlation has been commented upon by other researchers as well. ${ }^{9}$ The same correlation between p53 positivity and lymph node mets was found to be statistically significant in oral cavity and oropharyngeal cases in concordance with the work done by researchers like Jain A et al and Li Y et al. ${ }^{13,14}$ However, Takes RP et al records an opinion about this correlation which is diametrically opposite. ${ }^{18}$ The significance of $p 53$ in having a relationship with lymph node metastasis is the most important prognostic factor for overall survival of HNSCC as about two-third of the patients with HNSCC who present with lymph node metastasis have a poor five year survival rates of $40-50 \% .^{1}$ Thus, 553 expression can predict the overall prognostication in all patients of HNSCC.

Although p53 immunoexpression was seen to be in significant numbers in cases having vascular, muscular and perineural invasion, no significant correlation could be elicited statistically. Similar results were obtained by researchers like Yan JJ et al, who did not find any correlation between p53 overexpression and vascular invasion and early local recurrence. ${ }^{19}$ In the present study, majority of the cases were stage 4 (pT4) followed by stage 3 (pT3) and stage 2 (pT2). This correlation was found to be statistically significant $(\mathrm{p}=0.009$, chi square test $)$.

\section{CONCLUSION}

p53 immunoexpression when performed in a small Indian cohort of HNSCC patients showed that p53 was an important independent variable of prognostication with its expression and intensity increasing with tumor grade, lymph node metastasis and pathological stage. Similar results were obtained in oral cavity and oral pharyngeal cancers as well. However the study did not find such correlations in laryngeal and nasopharyngeal cases which could be attributed to small sample size in these two locations.

It is recommended that $\mathrm{p} 53$ should be employed in all the cases of HNSCC to predict prognosis and to comment upon eventual aggressiveness/tumor free survival. All the cases exhibiting p53 immunoexpression with higher intensity should be managed aggressively with both surgical and nonsurgical ancillary therapeutic means. This can be very useful while employing p53 on small punch biopsies and radical surgeries can be planned in a better elective manner after neoadjuvant therapy in p53 positive cases. Highly disfiguring radical surgeries can be avoided in cases of negative p53 immunoexpression and/or low staining intensity cases where excision and wait and watch policy can be adopted.

\section{Conflict of interest: None}

\section{REFERENCES}

1. Rousseau A, Badoual C. Head and Neck: Squamous cell carcinoma: an overview. Atlas Genet Cytogenet Oncol Haematol. 2012;16:14555. Crossref

2. Dragomir LP, Simionescu C, Margaritescu C, Stepan A, Dragomir IM, Popescu MR. P53, p16 and Ki67 immunoexpression in oral squamous carcinomas. Rom J Morphol Embryol. 2012;53:89-93. Crossref

3. Desai C. Squamous cell carcinoma of the head and neck region: Insights from the American Society of Clinical Oncology 2008 presentations. Indian J Cancer. 2008;45:90-2. Crossref

4. Mehrotra R, Singh M, Gupta RK, Singh M, Kapoor AK. Trends of prevalence and pathological spectrum of head and neck cancers in North India. Indian J Cancer. 2005;42:89-93. Crossref

5. Kumar A, Sharma A, Ahlawat B, Sharma S. Site specific effect of tobacco addiction in upper aerodigestive tract tumors: a retrospective clinicopathological study. Scientific World Journal. 2014;2014:460194. Crossref

6. Chitra G, Chandramouli A, Chanchal C. p53 mutations in head and neck squamous cell carcinoma. Int J Pharm Biomed Res. 2010;1:11721.

7. Kumar A, Sharma S, Ahlawat B, Sharma A, Prasad S, Chaudhary N. Synchronous Second Primary Upper Aerodigestive Tract Tumour Emphasizing Field Cancerization: A case report. J Med Res Prac. 2015;04:16-20.

8. Mannan R, Manjari M, Kaur H, Bhasin TS, Sharma S. Immunoexpression of p53 as an indicator of invasiveness in esophageal carcinoma:- a tertiary care hospital study from Punjab, India. Indian J Pathol Oncol. 2016;3;653-7. Crossref

9. Taneja K, Gupta S, Chauhan A, et al. Expression of p53 and bcl2 in squamous cell carcinoma of head and neck. Arch Clin Exp Surg. 2016;5:160-8. Crossref

10. Boslooper K, King-Yin Lam A, Gao J, Weinstein S, Johnson N. The clinicopathological roles of alpha-B-crystallin and p53 expression in patients with head and neck squamous cell carcinoma. Pathology. 2008;40:500-4. Crossref

11. Shenoi R, Devrukhkar V, Chaudhuri, Sharma BK, Sapre SB, Chikhale A. Demographic and clinical profile of oral squamous cell carcinoma patients: A retrospective study. Indian J Cancer. 2012;49:21-6. 


\section{Crossref}

12. Geisler SA, Olshan AF, Weissler MC, Cai J, Funkhouser WK, Smith $\mathrm{J}$ et al. p16 and p53 Protein expression as prognostic indicators of survival and disease recurrence from head and neck cancer. Clin Cancer Res. 2002;8:3445-50. Crossref

13. Li Y, Zhang J. Expression of mutant p53 in oral squamous cell carcinoma is correlated with the effectiveness of intra-arterial chemotherapy. Oncol Lett. 2015;10:2883-7. Crossref

14. Jain A, Maheshwari V, Mehdi G, Alam K, Sharma S. Diagnostic and prognostic significance of $\mathrm{p} 53$ protein expression in squamous cell lesions of the oral cavity. Int J Otorhinolaryngol. 2008;7:45-9.

15. Kerdpon D, Rich AM, Reade PC. Expression of p53 in oral mucosal hyperplasia, dysplasia and squamous cell carcinoma. Oral Dis. 1997;3:86-92. Crossref
16. Franzmann M, Buchwald C, Jacobsen G, Lindeberg H. Expression of p53 in normal nasal mucosa and in sinonasalpapillomas with and without associated carcinoma and the relation to human papillomavirus (HPV). Cancer Lett. 1998;128:161-4. Crossref

17. Ashraf MJ, Maghbul M, Azarpira N, Khademi B. Expression of Ki67 and p53 in primary squamous cell carcinoma of the larynx. Indian J Pathol Microbiol. 2010;53:661-5. Crossref

18. Takes R, Jong RJ, Alles MJ, Meeuwis CA, Marres HA, Knegt PP et al. Markers for Nodal Metastasis in Head and Neck Squamous Cell Cancer. Arch Otolaryngol Head Neck Surg. 2002;128:512-8. Crossref

19. Yan JJ, Tzeng CC, Jin YT. Overexpression of $\mathrm{p} 53$ protein in squamous cell carcinoma of buccal mucosa and tongue in Taiwan: an immunohistochemical and clinicopathological study. J Oral Pathol Med. 1996;25:55-9. Crossref 